\title{
Detecting and Georegistering Moving Ground Targets in Airborne QuickSAR via Keystoning and Multiple-Phase Center Interferometry
}

\author{
P. K. Sanyal, ${ }^{1}$ D. M. Zasada, ${ }^{1}$ and R. P. Perry ${ }^{2}$ \\ ${ }^{1}$ The MITRE Corporation, 26 Electronic Parkway, Rome, NY 13441, USA \\ ${ }^{2}$ The MITRE Corporation, 202 Burlington Road, Route 62, Bedford, MA 01730-1420, USA
}

Correspondence should be addressed to P. K. Sanyal, psanyal@mitre.org

Received 29 March 2007; Revised 24 August 2007; Accepted 16 November 2007

Recommended by Frank Ehlers

SAR images experience significant range walk and, without some form of motion compensation, can be quite blurred. The MITREdeveloped Keystone formatting simultaneously and automatically compensates for range walk due to the radial velocity component of each moving target, independent of the number of targets or the value of each target's radial velocity with respect to the ground. Target radial motion also causes moving targets in synthetic aperture radar images to appear at locations offset from their true instantaneous locations on the ground. In a multichannel radar, the interferometric phase values associated with all nonmoving points on the ground appear as a continuum of phase differences while the moving targets appear as interferometric phase discontinuities. By multiple threshold comparisons and grouping of pixels within the intensity and the phase images, we show that it is possible to reliably detect and accurately georegister moving targets within short-duration SAR (QuickSAR) images.

Copyright (c) 2008 P. K. Sanyal et al. This is an open access article distributed under the Creative Commons Attribution License, which permits unrestricted use, distribution, and reproduction in any medium, provided the original work is properly cited.

\section{INTRODUCTION}

Recently, synthetic aperture radar (SAR) imaging has become a common means of surveillance of the ground from airborne radars. Well-executed SAR images often produce images that are close to optical images wherein many ground features, such as buildings, roads, and so forth, as well as objects, such as trucks, cars, and so forth, can be recognized.

In many tactical applications, one is interested in detecting moving objects on the ground. SAR, by its nature, is a "still" picture and does not reveal directly which objects appearing in the image are moving. However, by comparing phases of SAR images from two channels of a multichannel radar, it is possible to detect moving objects.

This paper describes a phase interferometry technique for detecting moving targets in SAR and includes results using real data. Section 2 discusses the Keystoning technique and the acceleration correction used to produce fairly wellfocused, short-integration-time QuickSARs. The phase interferometry technique is discussed in Section 3. Moving targets in SAR appear in displaced positions. Once the moving targets are detected, they can be moved back to their actual locations in the SAR, that is, they can be georegistered. This is discussed in Section 4. Moving targets can be detected via phase interferometry when they are much stronger than the noise and ground clutter. In Section 5, we discuss a direct channel-to-channel clutter cancellation technique that cancels ground clutter by a significant amount, enhancing the detectability of the moving targets. Finally, Section 6 summarizes the paper.

\section{KEYSTONING AND ACCELERATION CORRECTION}

Without motion compensation, SAR images of the ground are generally blurred [1]. In 1997, MITRE reported the development of a technique called the Keystone process [2] for removing the range migration caused by the radial velocity component of each pixel's movement within the scene, whether moving or stationary with respect to the ground. (See the appendix for a short derivation of the Keystone process.)

The Keystone process removes the range migration effect of the constant radial velocity component of each pixel's 


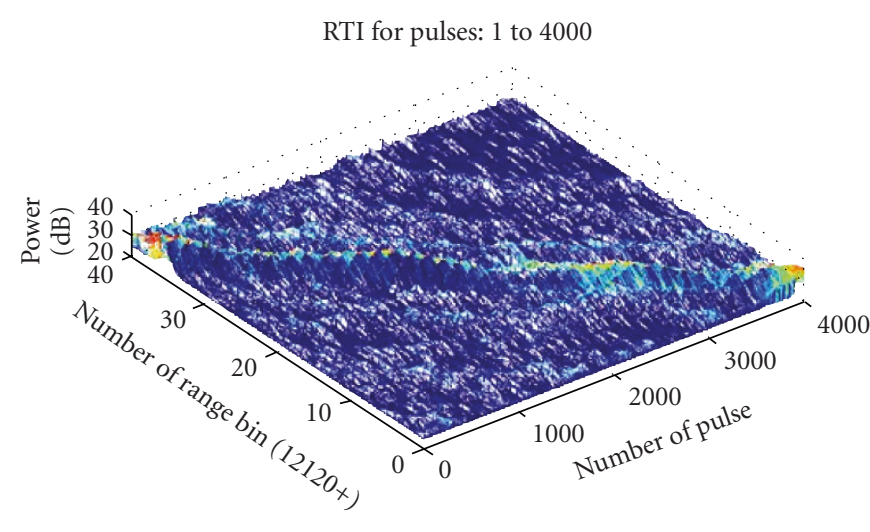

(a)

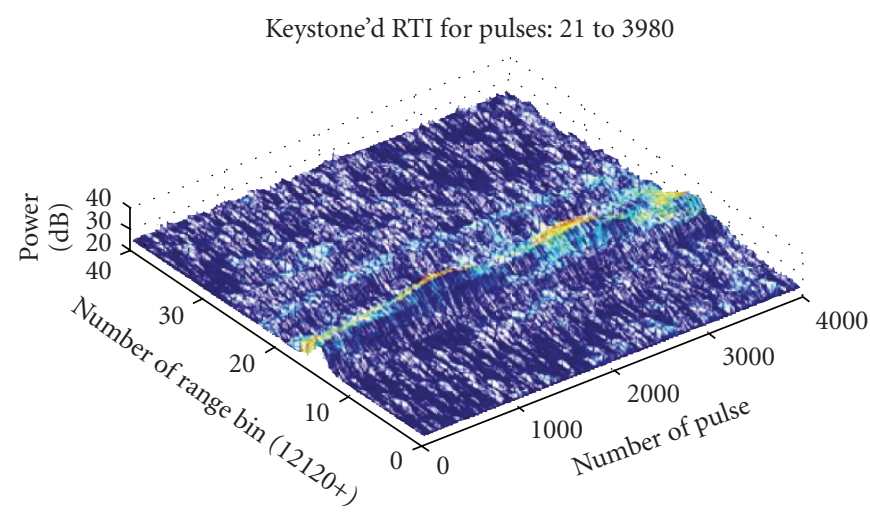

(c)

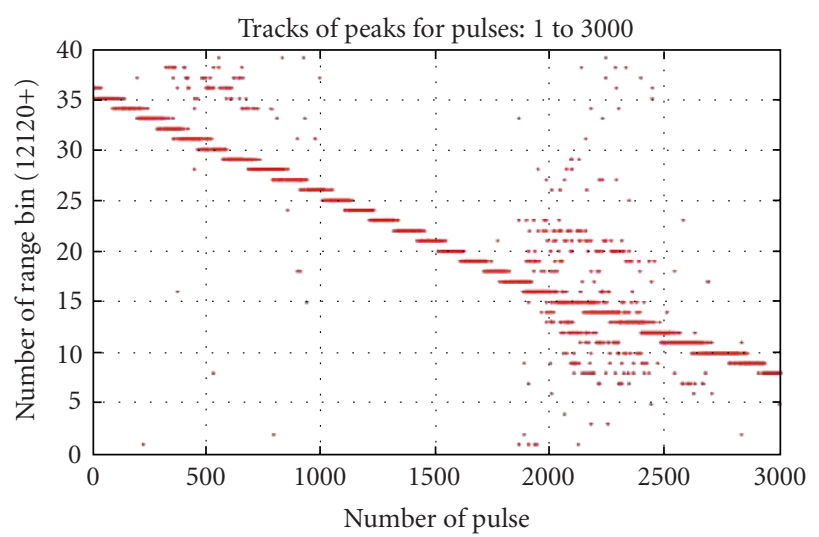

(b)

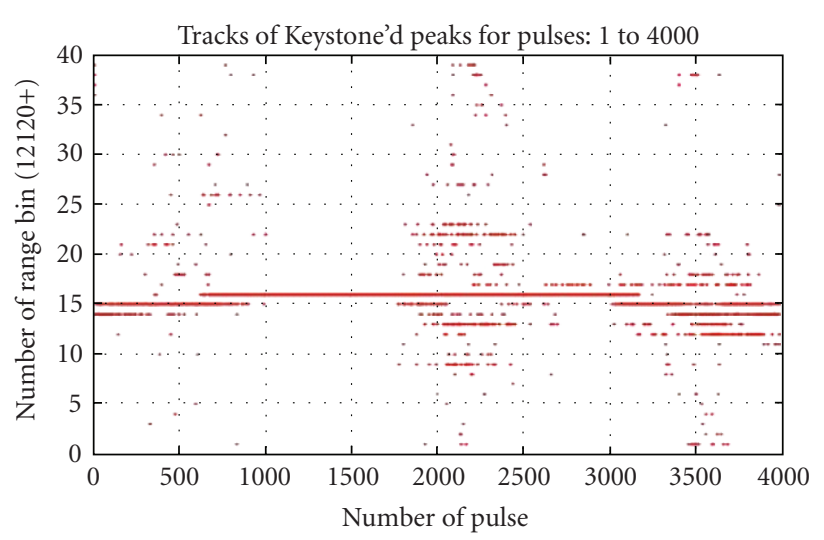

(d)

Figure 1: Keystoning removes range walk (top: before Keystoning; bottom: after Keystoning).

motion relative to the platform, whether of the ground itself or from moving targets embedded within the scene.

Figure 1 shows the effect of Keystoning on the range walk in a real SAR data collection. The left insets show the rangetime-intensity (RTI) plots while the right insets show the migration of the peaks in the RTIs over 2 seconds (4000 pulses at a pulse repetition frequency of $2000 \mathrm{~Hz}$ ).

If only Keystone formatting was applied, the SAR image would only be partially focused due to the remaining uncompensated quadratic and the higher-order terms in the target range. Therefore, after the range walk correction, the SAR data also has to be compensated for at least the acceleration to produce reasonably well-focused images.

Even when the radar platform is moving at a constant velocity (in a straight line and at a fixed speed), a point on the ground experiences a significant pseudo-acceleration with respect to the phase center of the radar.

For a simplified first-order analysis, consider the twodimensional airborne radar and the ground target geometry (i.e., the $x-y$ plane contains the line of sight, LOS, and the velocity vector) shown in Figure 2. The platform-centered coordinate system has the $x$-axis along the longitudinal axis of the aircraft and $y$-axis is out the wing. The aircraft is moving at a velocity $v$ which is along the $x$-axis.

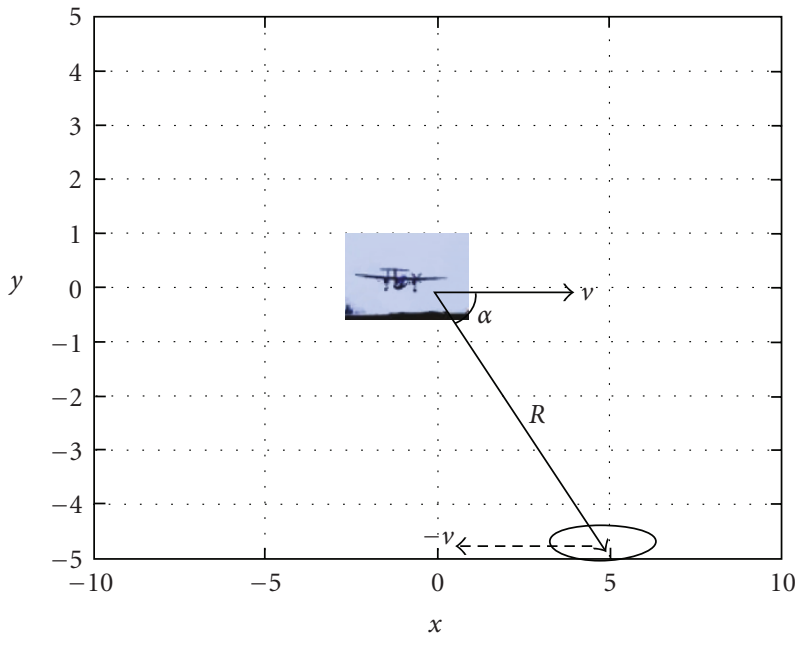

FIGURE 2: A typical radar-target geometry in SAR.

The range, $R$, of a point on the ground located at $(x, y$ axes) is

$$
R=\left(x^{2}+y^{2}\right)^{1 / 2} .
$$




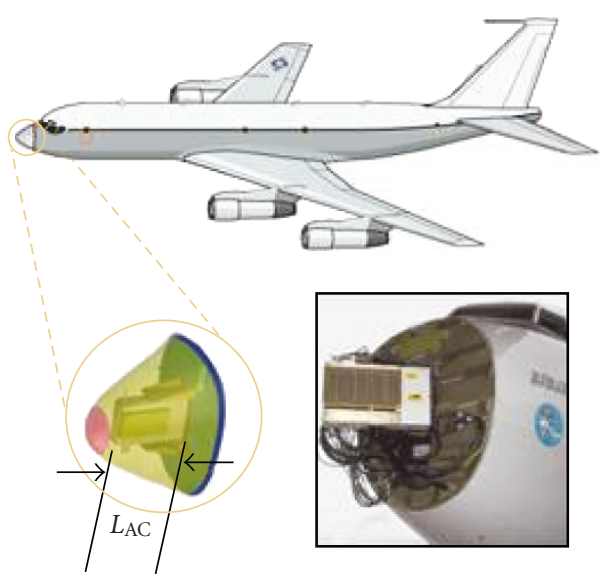

FIgURE 3: The 8-Channel X-band LiMIT system, mounted on a Boeing 707. $L_{\mathrm{AC}} \approx 18^{\prime \prime}$ (source: Lincoln Laboratory briefing, December 2004).

The radial velocity of the target is then

$$
\dot{R}=\frac{x \dot{x}+y \dot{y}}{\sqrt{\left(x^{2}+y^{2}\right)}}=\frac{x \dot{x}+y \dot{y}}{R},
$$

for the ground-fixed points, $\dot{y}=0$, and $\dot{x}=-v$. The range rate is then

$$
\dot{R}=-v \frac{x}{R}=-v \cos (\alpha),
$$

where $\alpha$ is the angle between the velocity vector and the LOS. The radial acceleration is then

$$
\begin{aligned}
\ddot{R} & =-v\left[\frac{\dot{x}}{\sqrt{\left(x^{2}+y^{2}\right)}}-x \frac{x \dot{x}}{\left(\sqrt{\left(x^{2}+y^{2}\right)}\right)^{3}}\right] \\
& =\frac{-v^{2}}{R}+\frac{v^{2} x^{2}}{R^{3}}=\frac{v^{2}}{R}\left(\cos ^{2}(\alpha)-1\right) .
\end{aligned}
$$

For the Lincoln Multimission ISR Testbed (LiMIT, 8 separate subapertures with receivers, operating frequency 9.2 $\mathrm{GHz}$, bandwidth $180-\mathrm{MHz}$, see Figure 3) data discussed here, $R=\sim 22 \mathrm{~km}, V=\sim 208 \mathrm{~m} / \mathrm{s}$, and the ground acceleration approaches $2 \mathrm{~m} / \mathrm{s}^{2}$ at $\alpha=90^{\circ}$, that is, at broadside. This acceleration has considerable effect on the focusing of the SAR image as can be seen in Figure 4 .

Figure 4 shows a single-channel SAR image resulting from the RTI shown in Figure 1. (LiMIT SAR data from Fort Huachuca, Ariz has data from eight subapertures or channels recorded separately; except for the spatial separations, all channels are nominally identical.) In this image, only the Keystone formatting has been applied but no acceleration correction has been performed yet.

Figure 5 shows the variation of the maximum of SAR image as a function of the acceleration correction applied. It is seen that for this image the image maximum increases by almost $16 \mathrm{~dB}$ from zero correction to the optimum acceleration correction of $-2.225 \mathrm{~m} / \mathrm{s}^{2}$.

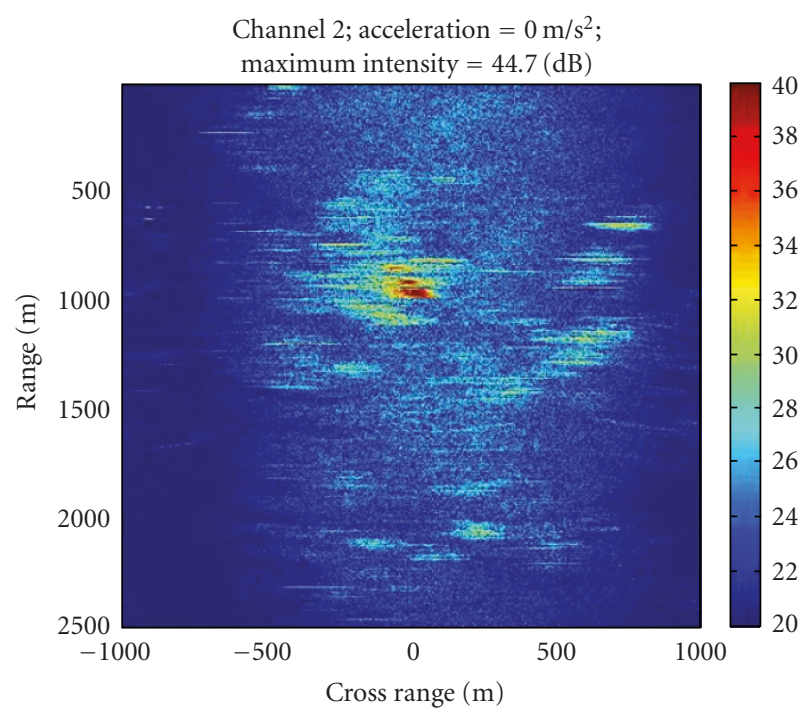

Figure 4: A LiMIT image with Keystoning but without any acceleration correction.

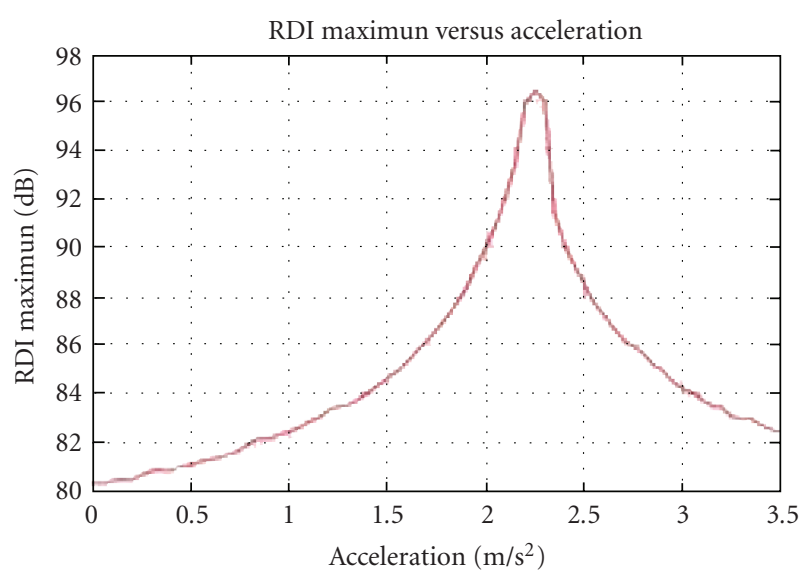

FIGURE 5: SAR maximum versus acceleration correction applied to an image.

Figure 6 shows the image with the optimum acceleration correction, along with the optical image for the corresponding area as available from Google. Note that the two axes of the image are shown as "range" and the "cross-range," with both units in meters. The cross-range values are obtained by properly scaling the computed Doppler values. In subsequent images, we have shown the cross-range dimension as Doppler, which retains the same essential information but without scaling to meters.

The degree of correspondence between the QuickSAR image and the optical image is excellent and the major features of the QuickSAR image are readily matched to those in the optical image.

Since each moving target in the scene may have a different acceleration, the moving targets can be individually further focused, which would improve their detectability. However, we have found that adequate detection is possible without the fine focusing of each target separately. We have used postdetection focusing of a target for identification purposes. 


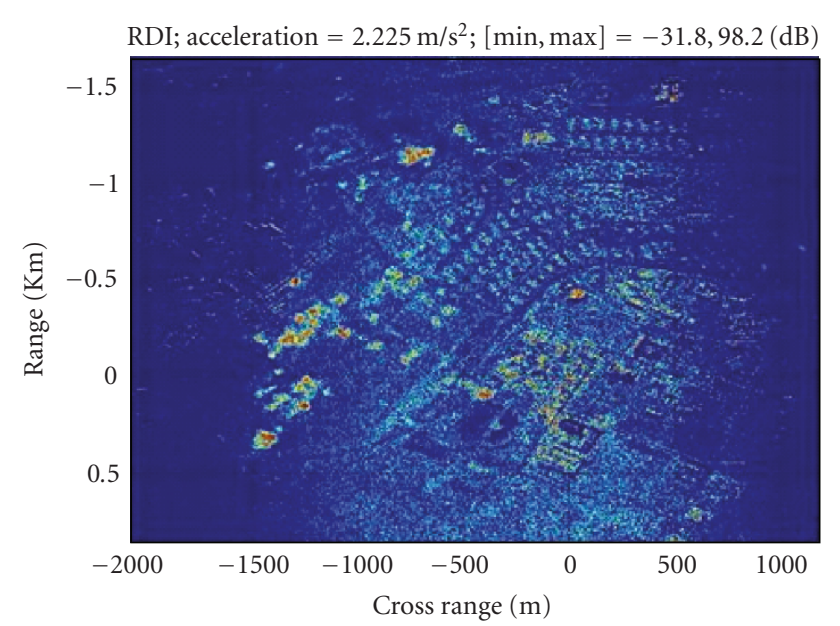

(a)

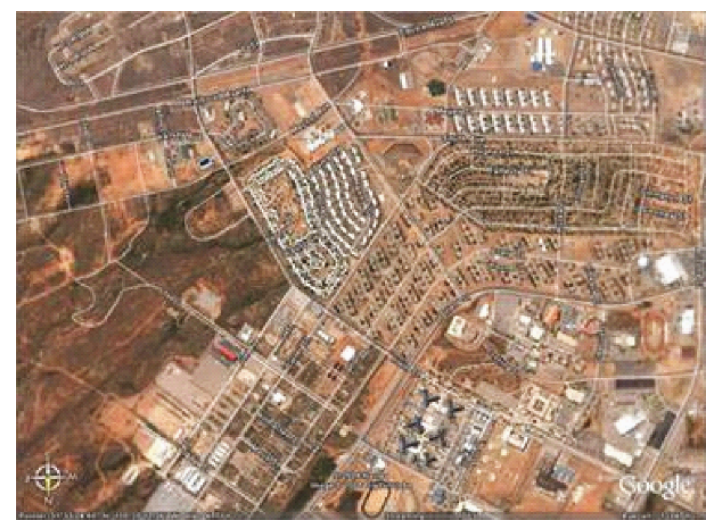

(b)

FIGURE 6: The Acceleration-corrected QuickSAR and the satellite optical image (source: Google).

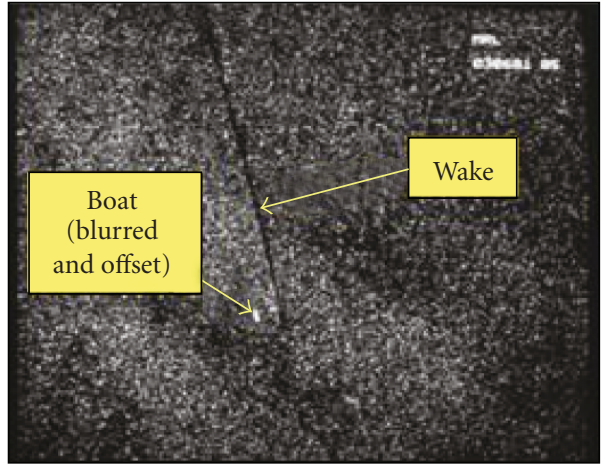

FIGURE 7: Boat-off-the-wake (source: www.sandia.gov).

\section{MOVING TARGET DETECTION WITH PHASE INTERFEROMETRY IN QUICKSAR}

The process described above produces fairly well-focused SAR images. Now, given a focused SAR image, how does one know if any of the detectable objects in the SAR are moving objects? This can be done via "phase interferometry."

The target motion causes the moving targets to appear at locations different from their true instantaneous locations on the ground in the SAR image. This is due to the coupling of the cross-range position to the target radial velocity and the fact that the moving target and the ground under it have different radial velocities relative to the platform. The result is the well-known "train-off-the-track" where the moving train appears to be hovering off the image of the track, or the "boat-off-the-wake" phenomenon (see Figure 7) where the moving boat is clearly off the tip of the boat wake, where it really belongs.

\subsection{Shift in cross-range due to Doppler processing}

How far off is the moving object from its actual location in a SAR? In the simplified 2D geometry considered above, the
Doppler, $f_{D}$, of a ground point at a small angle $\theta$ radians from the normal to the radar velocity vector is given by

$$
f_{D}=\frac{2 V \theta}{\lambda} .
$$

If a moving target has the Doppler $f_{\text {targ }}$, then it will appear shifted in cross-range by an angle $\theta$ such that

$$
f_{\mathrm{targ}}=\frac{2 V \theta}{\lambda}, \quad \frac{2 v_{\mathrm{targ}}}{\lambda}=\frac{2 V \theta}{\lambda}, \quad \text { or } \quad \theta=\frac{v_{\mathrm{targ}}}{V} .
$$

At a range $R$, this amounts to a linear shift in cross-range,

$$
\text { CrossRangeShift }=R \theta=\frac{R v_{\text {targ }}}{V} .
$$

For the SAR image in Figure 6, we have $R \approx 22 \mathrm{~km}$, platform $V \approx 208 \mathrm{~m} / \mathrm{s}$. If the target radial velocity $v_{\text {targ }}=30 \mathrm{~m} / \mathrm{s}$ $(\approx 67.5 \mathrm{mph})$,

$$
\text { CrossRangeShift } \approx \frac{22 * 10^{3} * 30}{208}=3.173 \mathrm{~km} .
$$

The upper inset of Figure 8 shows a portion of the SAR image of the urban area of Fort Huachuca, Ariz. In this QuickSAR image (2-second integration), one can easily discern the roads, buildings, and other features of reasonably large size. There are also other easily detectible, bright objects. Is there a way to know if any of these other objects are moving?

\subsection{Phase image}

To make use of the above-mentioned cross-range displacement phenomenon to detect moving targets in SAR images, we form a phase-interferometry image as shown in the lower inset of Figure 8. It is the color-coded plot of the pixel-topixel phase difference between channels one and eight. (The two end channels of the 8-channel LiMIT system are chosen 


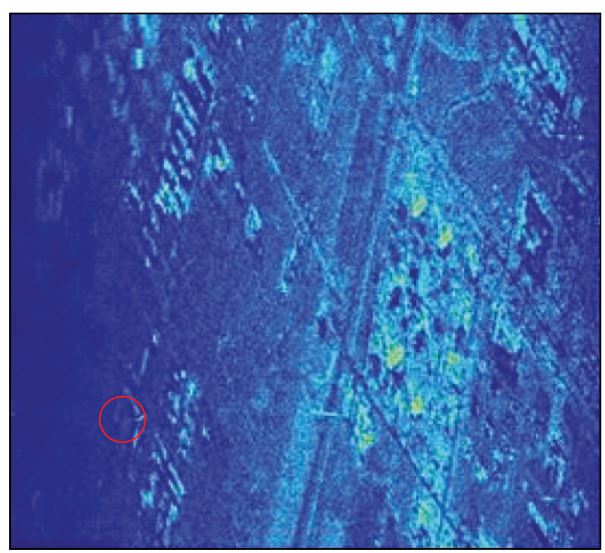

(a) SAR image from channel 1

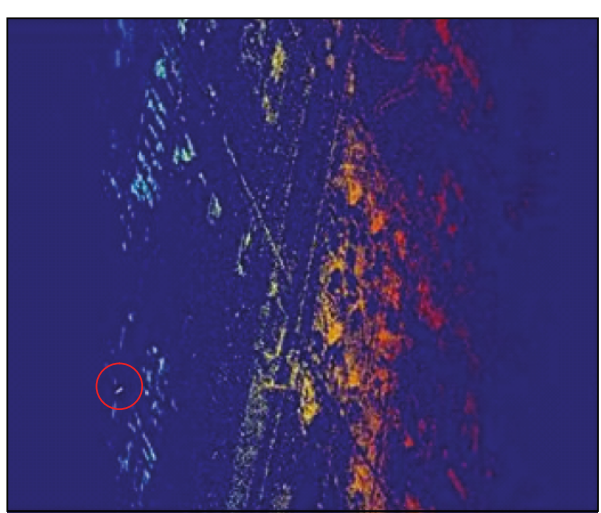

(b) Phase-difference image using channelsland 8

FIgURE 8: SAR and phase-difference images of Fort Huachuca.

to enhance the phase difference.) The phase difference values vary between $-\pi$ and $+\pi$, with red for $+\pi$, gradually changing to blue for $-\pi$.

In such an interferometric phase image, all points on the ground should nominally appear as a continuum of phase differences from left to right along the cross-range axis. In Figure 8, we see the fringes parallel to the range direction gradually changing in color along the cross-range direction.

Pixels of low intensity usually consist of system noise rather than ground-returns, and hence the channel-tochannel phase difference becomes unrelated to the indicated location on the ground and can randomly vary between $-\pi$ and $+\pi$ and the phase-difference image becomes filled with speckles.

We have arbitrarily set the phase difference values for pixels in the SAR which are below a selected $\mathrm{dB}$ value (here, it is $10 \mathrm{~dB}$ ) to $-\pi$ with the purpose of reducing speckles. Setting the above LiMIT reduces the speckles. It also helps to delineate areas of low reflectivity, and hence the same features we see in the SAR (see Figure 8(a)), are also visible in the phasedifference image (see Figure 8(b)).

Now, while all the points on the ground appear as a continuum of phase differences along the cross-range axis, the moving targets appear as discontinuities because a pixel that represents a moving object appears displaced from the ground point on which it is instantly situated. This causes a phase discontinuity. In the color-coded phase difference image, the phase discontinuity shows up as a color anomaly. By searching for color discontinuities, one can easily identify several moving targets in the interferometric image. One such target is circled in red. Thus, the phase-interferometry image helps to indicate which detected objects in the SAR are moving objects [5-7]. The color anomaly may not be obvious in this highly shrunk image but is quite clear in an exploded version of the image.

\subsection{Automated phase discontinuity detection}

A trained operator would be able to detect moving targets in a color-coded phase difference image as described above. For

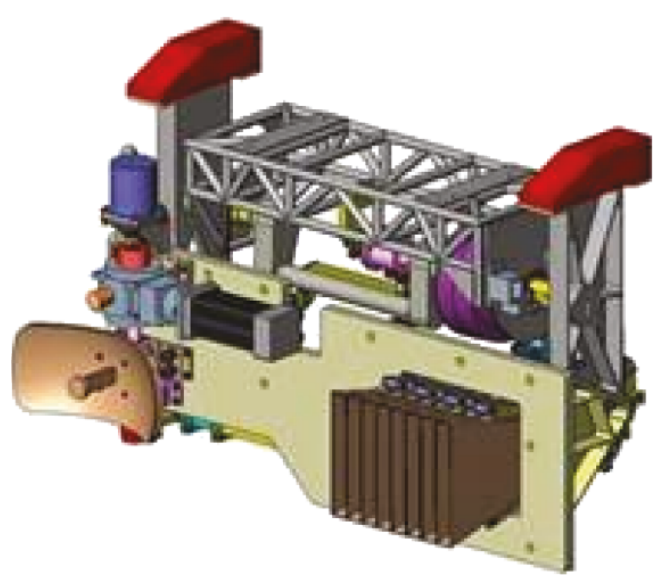

Figure 9: The General Dynamics Convair 580 belly-mounted $\mathrm{X}$-band DCS system with one transmit antenna and eight receive horns.

automated moving target detection, we execute the following steps:

(a) apply an appropriate acceleration correction to focus the ground plane;

(b) fit a reference plane, in a least-square error sense, to the phase difference image;

(c) look for pixels whose phase differences deviation from the reference plane exceeds a given threshold;

(d) from the above, select those that also exceed an amplitude threshold.

This technique is illustrated with results from another dataset. This is the General Dynamics DCS 8-Channel, 160MHz-bandwidth X-band data from Eglin, Fla (see Figure 9). In this scenario, there were seven targets moving in circles on the runway, and the GPS data from the vehicles as well as the motion data from the radar platform are available.

The top inset in Figure 10 shows a QuickSAR (coherent processing interval $(\mathrm{CPI})=0.83$ second) of the portion of the runway where the targets were presented. 


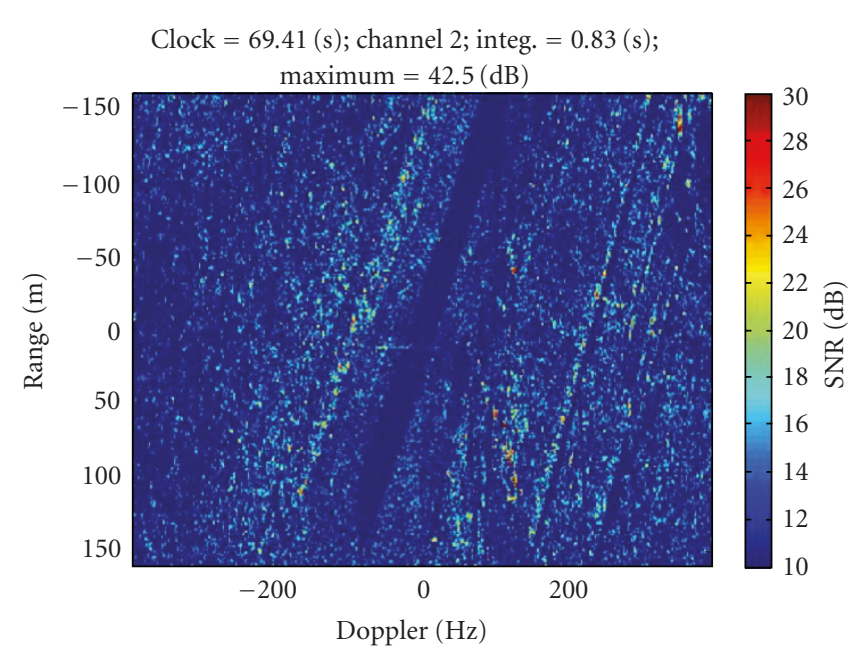

(a)

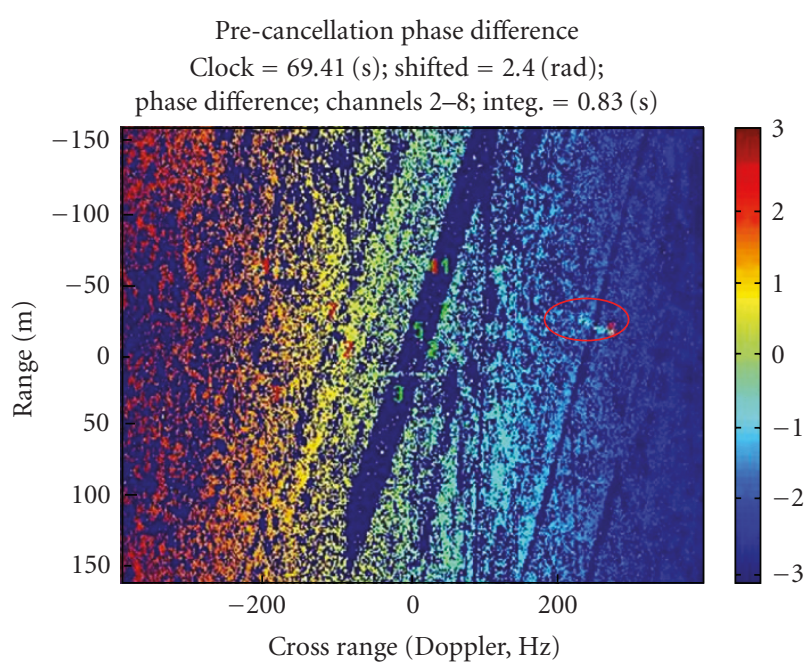

(b)

Figure 10: QuickSAR and Phase-difference images of the Eglin Runway.

This QuickSAR is generated from the channel 2 data of the 8-channel dataset. (Note that the cross-range axis is labeled as Doppler in Hz; we did not scale it to meters or azimuth angle, but the same information is retained.)

The bottom inset in Figure 10 is the phase-difference image using channels 2 and 5 . Note that the runway is still clearly discernable. The numbers in green indicate the actual positions of the moving targets at the time the data was collected, while the displaced red numbers indicate where they should appear in the range-Doppler SAR image. It is interesting to note that target number 5, which is a large target, shows up more prominently in the phase-difference image (red circle) than in the QuickSAR image (the color anomaly is much clearer in a larger display of the image). This visual observability is very useful to a human operator because he/she can easily place the detections in the "context" of the background terrain, that is, whether it is near a building, on a road, and so forth. Below, we describe how a machine detects the moving targets.

The blue dots in the upper left inset in Figure 11 are the row-by-row plots of the phase differences shown in Figure 10. The red dots (which appear as a line because of the compactness) are the phases from the plane fitted to the actual phase differences.

Ideally, all points on the ground would have phase differences that lie on a plane and only moving targets will have phase differences that deviate from the plane. But in reality, because of the system noise, variations in terrain elevation, and so forth; the actual phase differences, of course, do not all lie on the plane.

The smaller the clutter power is in a pixel without a target in it, the more likely it is to be affected by the system noise and thus more likely to deviate from the plane. The upper right inset of Figure 11 shows that a very large number of pixels exceed the selected 1-radian phase deviation threshold but a significant number of them are below $15 \mathrm{~dB}$. On the other hand, the pixels corresponding to the moving targets, we expect to detect, should be of sufficient strength as well as deviate significantly from the phase plane. Thus, the moving targets have to meet the dual thresholds: one in power and the other in phase deviation [8]. If the power threshold is set at $15 \mathrm{~dB}$ in this case, the number of pixels that deviate from the plane by more than the 1-radian phase deviation threshold drops to 85, as shown in the lower left inset of Figure 11.

The lower right inset in Figure 11 shows these pixels as the detected targets. These are indicated with red $x$ 's. In this particular image, there were as many as 85 detections. While some of these detections are likely to be false alarms, most of them are possibly multiple detections from "extended," that is, large targets.

\subsection{Clustering of the detections}

An observer can readily group many of these detections into possible clusters that belong to the same targets. We have used an automatic clustering algorithm from Matlab, which results in eight clusters in this image. These clusters are depicted with red circles. Note that the circles only indicate the center of the clustered detections; they do not indicate which detections belong to the cluster or how many detections were included in the cluster.

Notice that one cluster coincides with the expected location of target number 2, two clusters appear to coincide with target number 1, four clusters appear to coincide with target number 5 (which is known to be a large target), and the eighth cluster appears to comprise one or more false alarms.

A human observer examining the four clusters near target number 5 or the two near target number 1 would have readily combined them into single clusters each. The Matlab fuzzylogic clustering algorithm used could also possibly have been tweaked to yield similar results but we did not attempt to do so at this time.

Zhang et al. also use a dual threshold technique [9] for detecting slow moving targets in along-track interferometric 


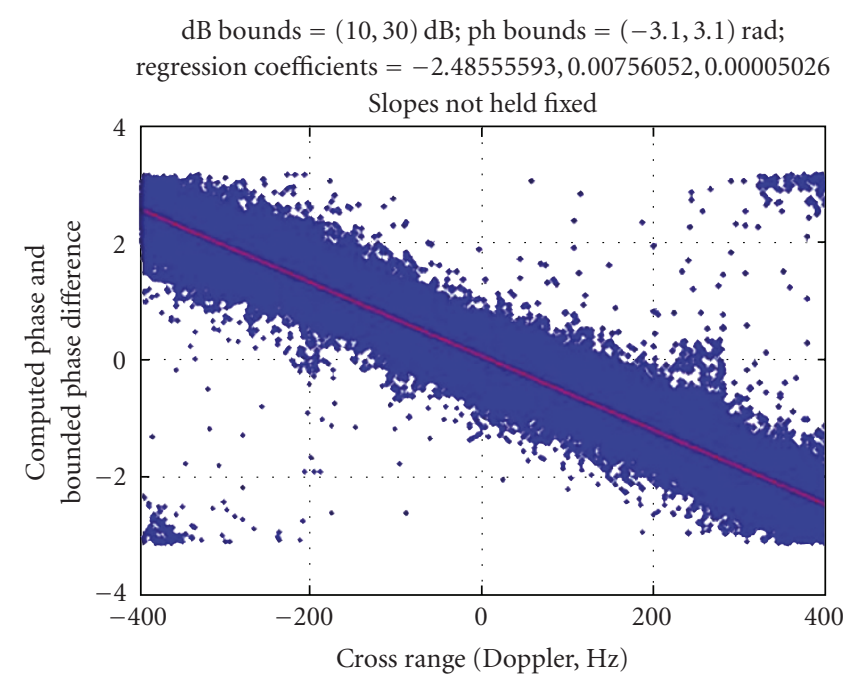

(a)

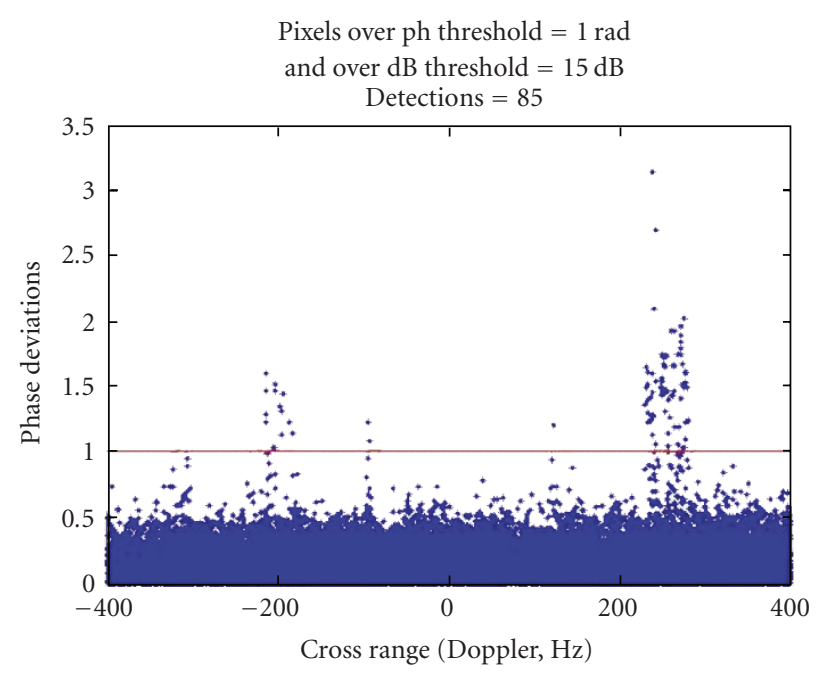

(c)

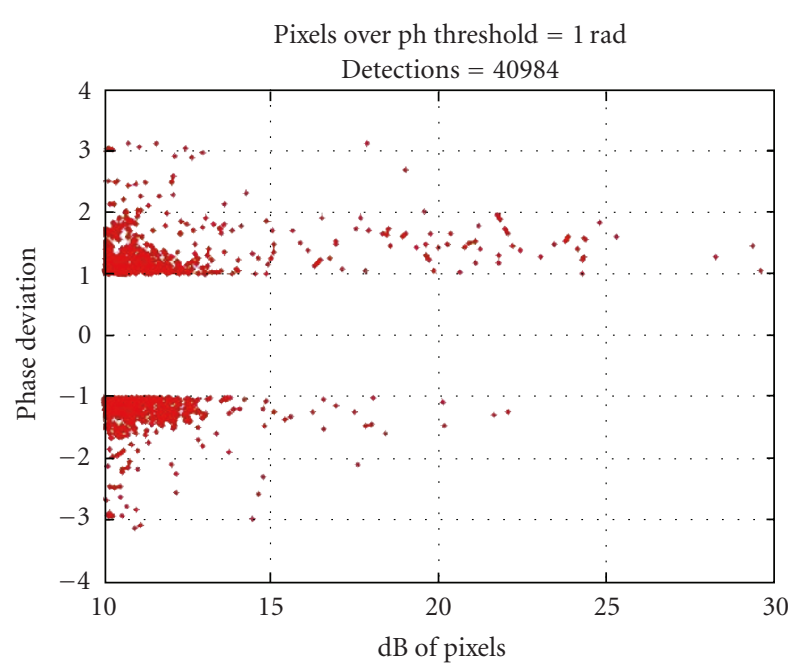

(b)

85 dets; 8 clusters; ph threshold $=1$ (rad); $\mathrm{dB}$ threshold $=15(\mathrm{~dB})$; clock $=69.41(\mathrm{~s})$; channel 2 ; integ. $=0.83(\mathrm{~s})$; maximum $=42.5(\mathrm{~dB})$

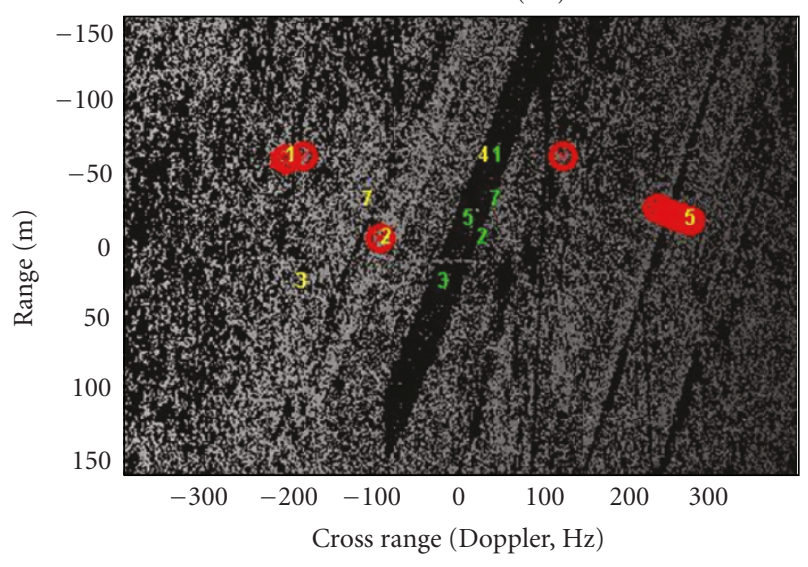

(d)

FIGURE 11: Machine detection of moving targets in a real QuickSAR.

SAR (ATI-SAR). They present results using L-band data from Jet Propulsion Laboratory (JPL) AirSAR data and show the successful detection of the breaking waves in the Monterey Bay area of California. A major difference between the two methods is that while they talk about a "phase calibration" which may be affected by the aircraft crab angle, we perform a least-square error estimation of the "phase-difference plane" and do not worry about the minute aircraft motions.

Further, the interferometric technique described here leads to the direct channel-to-channel clutter cancellation technique for detecting weaker moving targets.

\section{GEOREGISTRATION OF DETECTED MOVING TARGETS IN QUICKSAR}

The "apparent" locations of the moving targets detected in the SAR image are not their actual locations on the ground.
In fact, recall that the detection of the moving targets in the SAR image depends on the fact that they are displaced in cross-range from their actual locations. Therefore, after detection, they need to be "georegistered," that is, they need to be placed correctly at their actual locations within the SAR image. In many applications, a target that is properly georegistered within the SAR has much more intuitive positional information than the position derived from the range and angle and converted to latitude/longitude.

Fortunately, the georegistration is almost a byproduct of the detection process. The top inset in Figure 12 shows the phase difference of the detected pixels (blue dots) and the plane fitted to the phase differences of all the ground points (represented by red dots, which have merged to appear as a line).

Since the moving targets are also on the ground (the given assumption), the actual locations of the detected pixels 


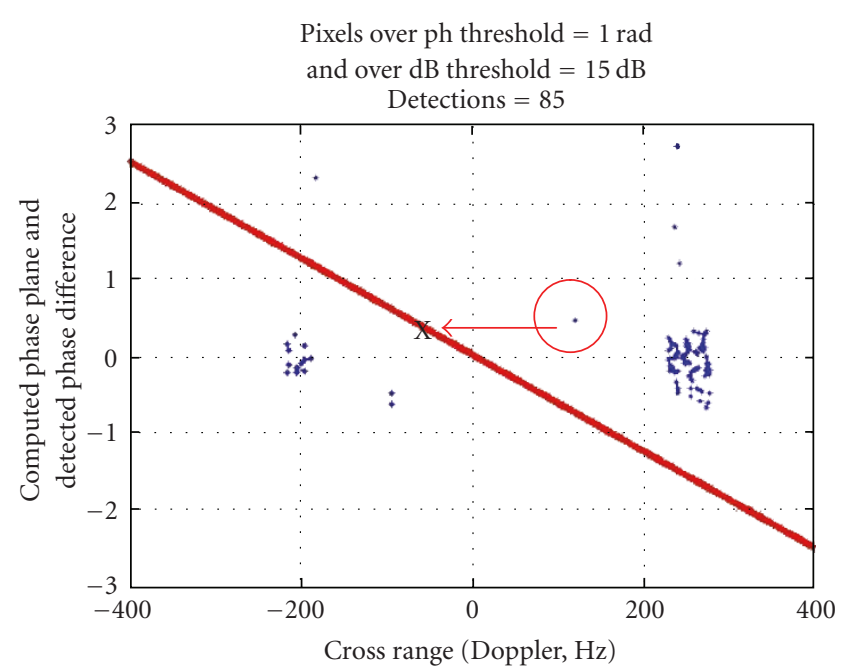

(a)

Geo-registered; weighted cluster average; ph threshold = $1(\mathrm{rad})$; $\mathrm{dB}$ threshold $=15(\mathrm{~dB})$; clock $=69.41(\mathrm{~s})$; channel 2; integ. $=0.83(\mathrm{~s}) ;$ maximum $=42.5(\mathrm{~dB})$

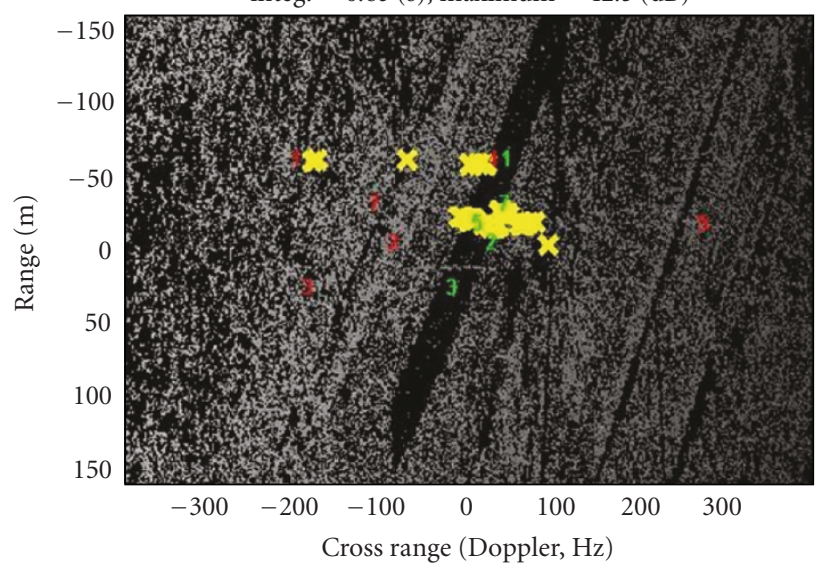

(b)

FIGURE 12: Geo-registration of moving targets in a real QuickSAR.

are found by displacing them in cross-range until they lie on the red line, as indicated in the bottom inset of Figure 12.

This displacement may be done on a detection-bydetection basis or a cluster basis. In a detection-by-detectionbasis georegistration, each detection is moved independently. As may be obvious, because of the various amounts of noise in the individual pixels, the pixels that appear to be in a cluster before georegistration can become scattered after the above-described displacement.

\subsection{Cluster move}

Since a clustering has already been performed, it is easy to move all the detections in a cluster together to appear as the same cluster after the georegistration. For this, one computes the average phase difference for the cluster and uses this value to relocate the whole cluster on the red line.

A slight twist on the above technique is to find a weighted average of the phase difference using the pixel power to weight the phase difference values. This plausibly reduces the effect of low-power pixels which are likely to be noisier.

The right inset in Figure 12 shows the geolocated detections using the weighted average method. It is seen that the majority of the detections are now appearing on the runway where they actually belong.

\subsection{Weighted cluster move}

Here, we have shown only one image 0.83 -second frame of the dataset from this SAR data collection scenario which lasts for about 200 seconds. We have applied this technique to the 250 or so frames from this dataset and have obtained consistent detection and georegistration results.

\section{DIRECT CHANNEL-TO-CHANNEL CLUTTER CANCELLATION (DC4) FOR MOVING TARGET DETECTION}

The phase-interferometry technique described above works well when the target return is strong and well above noise and ground clutter. In the above examples, we were able to see the target in the SAR with the naked eye and then used the phase interferometry to determine if they were moving.

If the moving target return is not significantly stronger than the ground clutter in that pixel, it will not be noticed in the phase difference image either because the phase difference will not be as strongly influenced by the target return. Clearly, cancelling the clutter will enhance the moving target detection.

In an ideal case, that is, channels are perfectly balanced, there is no system noise, there are no moving targets, there is no internal clutter motion, and so forth, the SAR images from any two channels only differ in phase that varies linearly with cross-range or equivalently with Doppler. This phase difference can be found by simply differencing the two complex images. If all the phase difference values were plotted against the Doppler, one would see a straight line. And if one of the images is appropriately weighted by this phase difference matrix and subtracted from the other, it should result in a null image, that is, an infinite (in $\mathrm{dB}$ ) clutter cancellation. Moving targets, if present, will suffer a cancellation loss but will not be cancelled completely. The moving target return, though partly diminished, will be detected with ease.

In a real case, there are system and other sources of noise. And the lower the clutter return is in a pixel, the more dominated it is by the noise. The upper left inset in Figure 11 is a plot of the real phase differences (lower insert in Figure 10) against the Doppler.

The actual distribution of the phase values is not shown in this plot but a linear trend is clearly visible and in the absence of any noise (from system or due to internal clutter motion), all phase differences would have collapsed on a straight line like the red line shown. Indeed, the red line is the plot of the phases from a plane fitted to the phase values in Figure 10.

This plane is derived as follows. Consider the phase difference image as an mxn matrix of phase difference numbers, 


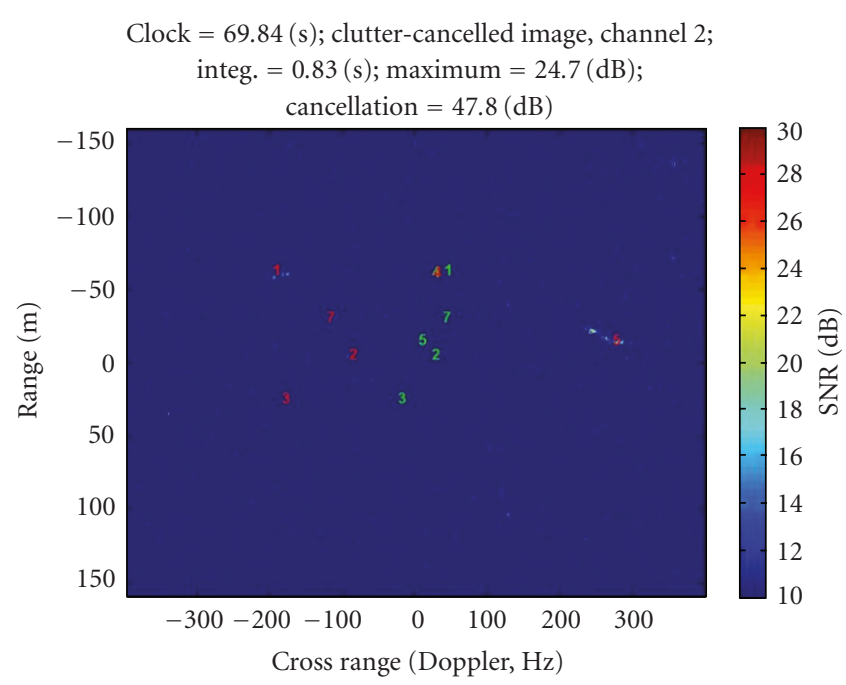

FIGURE 13: Clutter-cancelled SAR image.

where $m$ is the number of range cells and $n$ is the number of Doppler cells, that is,

$$
\begin{gathered}
\Delta \varphi(i, j), \\
\text { range cell } i=1, \ldots, m, \\
\text { Doppler cell } j=1, \ldots, n .
\end{gathered}
$$

We fit a plane to this observed surface:

$$
\Delta \varphi(i, j)=c_{0}+c_{\text {Doppler }} j+c_{\text {range }} i+\text { noise },
$$

where the c's are the regression coefficients. The phasedifference plane is primarily sloped in the Doppler dimension and, therefore, $c_{\text {Doppler }}$ will have a significant value, but we also allow the plane to be sloped in the range dimension and hence the coefficient $c_{\text {range }}$.

Having computed the regression coefficients estimates, we compute the "least-square-fitted" phase difference $\Delta \hat{\bar{\phi}}$. In Figure 11, the red line is the edge view of this fitted plane.

Let the complex images formed at the two channels, numbered 1 and 8 , be designated $I^{1}$ and $I^{8}$, respectively. Ideally, (i.e., in the absence of system noise and moving targets) the images $I^{1}$ and $I^{8}$ are simply phase-shifted versions of each other and given the phase shifts, one image can be used to cancel another. If we now subtract the image from channel 8 weighted by this smoothed phase from the channel 1 image, we get the clutter-cancelled image

$$
I^{1 / 8}=I^{1}-I^{8} \exp (j \Delta \hat{\bar{\phi}}) .
$$

Any channel may be cancelled by another channel using the above method. Figure 13 shows some results of enhancing moving target return by channel-to-channel cancellation of the ground clutter and in a channel number 2 image using the channel number 5 image. (The precancellation channel 2 image, not shown here, appears almost identical to channel 1 precancellation image in the top inset in Figure 10.) Com-

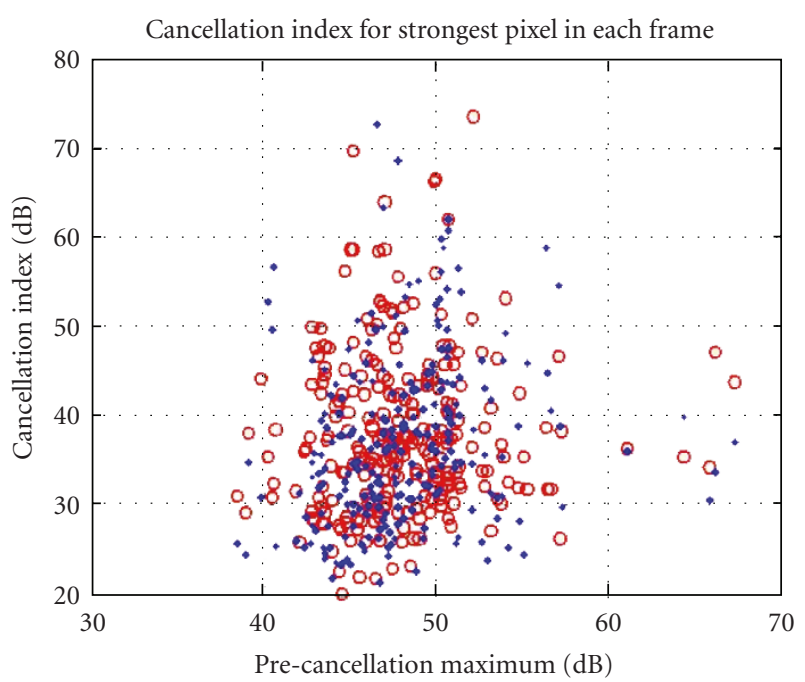

FIGURE 14: Measured QuickSAR clutter cancellation achieved with the General Dynamics data.

paring this image with the top inset in Figure 10, it appears that, as far as the naked eye can tell, almost all of the ground clutter has been cancelled.

The GD dataset mentioned above contains over $200 \mathrm{sec}-$ onds of a SAR data run. At about 0.83 seconds of integration time per QuickSAR, we generated about 250 frames. Each image was cropped to retain only the area of interest. The cropped image is 383 range cells $\times 661$ Doppler cells, thus containing over a quarter million pixels of various strengths. It is most likely that each pixel undergoes a different degree of cancellation and it is worth looking at all pixels to gain a more thorough picture of the cancellation achieved. However, for a quick analysis, we only looked at the strongest pixel from each frame.

We first found the strongest pixel prior to cancellation. After cancellation, we determine the strength of the same pixel (not necessarily the strongest in the postcancellation image) and compute the cancellation. Figure 14 plots the cancellation ratio that was obtained with this real data (the red circles are the cancellation values when channel number 2 is cancelled with channel number 5 , and the blue dots are the values when channel number 5 is cancelled with channel number 8 ).

Based on only the strongest pixel from each frame, the average cancellation over the 250 frames is about $37 \mathrm{~dB}$. As a reference, it is noted that Muehe and Labitt [10] mention a clutter-to-noise improvement factor (CIF) of $46 \mathrm{~dB}$ with the MARS radar developed by Lincoln Lab specifically for DPCA experiments.

We have not done a one-on-one comparison of this clutter cancellation technique with the widely used space time adaptive processing (STAP) technique $[11,12]$ for ground clutter cancellation yet. However, it may be noted that with STAP, one is often concerned about the non-Gaussianity and nonhomogeneity of the ground clutter. With the direct channel-to-channel clutter cancellation technique, those issues should not be of concern because each pixel from one channel is used to cancel the corresponding pixel from the 
other channel and, therefore, how the clutter strength varies from pixel to pixel is not a major concern.

\section{CONCLUSION}

In this paper, we have described an interferometric scheme for detecting and georegistering surface moving targets in multichannel SAR. The interferometric scheme described here is able to detect moving targets well within the ground clutter.

Since the detections take place within a SAR image, and further, they are properly georegistered, this has the additional benefits of "contextual" detection, i.e., the detections are already "in context." In other words, one can see if they are on roads, runways, near buildings, and so forth.

We have also described a novel channel-to-channel clutter cancellation technique that enhances the detectability of moving targets. It has not been exhaustively compared with the STAP technique but we note that it appears to suffer less from the non-Gaussian or nonhomogeneous character of ground clutter in many areas of interest, that is, urban areas, littoral regions, near forest lines, and so forth.

All the results included here are from real multichannel radar data that add to the credibility of the techniques.

\section{APPENDIX}

\section{KEYSTONE FORMATTING}

Keystone formatting can be derived by noting that the spectrum of a single-received pulse is given by

$$
S_{r}(f)=P(f) \exp \left[-i \frac{4 \pi}{c}\left(f+f_{0}\right) R(t)\right],
$$

where $P(f)=$ spectrum of transmitted pulse, $f=$ baseband frequency $(-B / 2 \leq f<B / 2), f_{0}=$ carrier frequency, $R(t)=$ range to target at time $t$.

Expanding $R(t)$ in a Taylor series, we get:

$$
R(t)=R\left(t_{0}\right)+\dot{R}\left(t_{0}\right) t+\frac{1}{2} \ddot{R}\left(t_{0}\right) t^{2}+\cdots .
$$

Substituting (A.2) into (A.1) and dropping cubic and higher-order terms,

$S_{r}(f)$

$=P(f) \exp \left[-i \frac{4 \pi}{c}\left(f+f_{0}\right) R-i \frac{4 \pi}{c}\left(f+f_{0}\right) \dot{R} t-i \frac{2 \pi}{c}\left(f+f_{0}\right) \ddot{R} t^{2}\right]$.

The second term in the brackets containing the product $f \dot{R} t$ gives rise to range walk. This term becomes zero when we use the temporal transformation $t=\left(f_{0} /\left(f+f_{0}\right)\right) t^{\prime}$.

With the above substitution, (A.3) can be written as

$S_{r}(f)$

$=P(f) \exp \left[-i \frac{4 \pi}{c}\left(f+f_{0}\right) R-i \frac{4 \pi}{c} f_{0} \dot{R} t^{\prime}-i \frac{2 \pi}{c}\left(f+f_{0}\right) \ddot{R}\left(\frac{f_{0} t^{\prime}}{f+f_{0}}\right)^{2}\right]$.

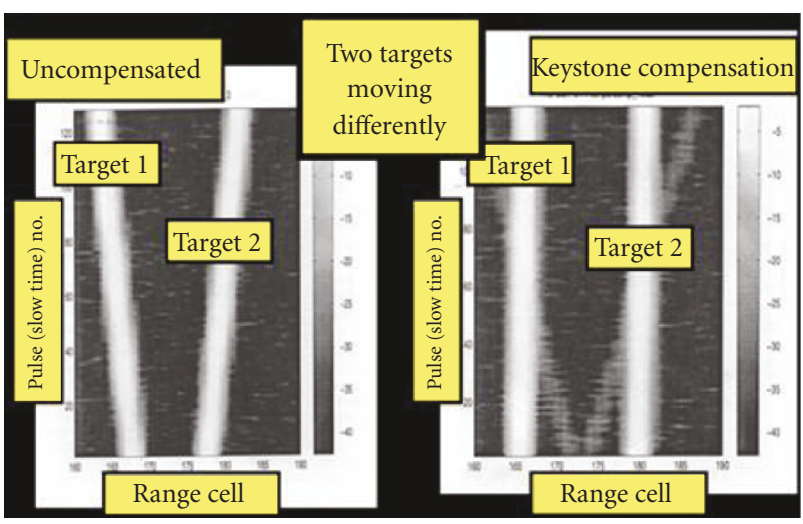

FIGURE 15: Keystone formatting performs range-walk correction for all targets moving at different velocities.

Since Keystone formatting does not solve the quadratic (or higher-order) motion problem, let us also drop the quadratic term in (A.4) and simplify it to

$$
S_{r}(f)=P(f)^{*} \exp \left[-i \frac{4 \pi}{c}\left(f+f_{0}\right) R-i \frac{4 \pi}{c} f_{0} \dot{R} t^{\prime}\right] .
$$

The Keystone process compensates for the different radial velocities of all moving targets simultaneously. Figure 15 shows the result of a first-order simulation to illustrate how targets moving at two different velocities are simultaneously corrected for range-walk.

\section{ACKNOWLEDGMENTS}

The authors would like to thank AFRL Rome Research Site in Rome, NY for providing them with the various real radar data sets used in their work. They would also like to thank the anonymous reviewers for their detailed critiques which helped to greatly improve this paper.

\section{REFERENCES}

[1] I. G. Cumming and F. H. Wong, Digital Processing of Synthetic Aperture Radar Data, Artech House, Boston, Mass, USA, 2005.

[2] R. P. Perry, R. C. DiPietro, and R. L. Fante, "SAR imaging of moving targets," IEEE Transactions on Aerospace and Electronic Systems, vol. 35, no. 1, pp. 188-199, 1999.

[3] R. P. Perry, R. C. DiPietro, and R. L. Fante, "Coherent integration with range migration using keystone formatting," in Proceedings of IEEE Conference on Radar (RADAR '07), pp. 863868, Waltham, Mass, USA, April 2007.

[4] E. F. Stockburger and D. N. Held, "Interferometric moving target imaging," in Proceedings of the IEEE International Radar Conference, pp. 438-443, Arlington, Va, USA, May 1995.

[5] P. K. Sanyal, R. P. Perry, and D. M. Zasada, "Detecting moving targets in SAR via keystoning and phase interferometry," in Proceedings of the 5th International Radar Symposium (IRSI '05), Bangalore, India, December 2005.

[6] P. K. Sanyal, D. M. Zasada, and R. P. Perry, "Detecting moving targets in SAR via keystoning and multiple phase center interferometry," in Proceedings of IEEE Conference on Radar (RADAR '06), pp. 498-503, Verona, NY, USA, April 2006. 
[7] D. M. Zasada, P. K. Sanyal, and R. P. Perry, "Detecting moving targets in clutter in airborne SAR via keystoning and multiple phase center interferometry," in Algorithms for Synthetic Aperture Radar Imagery XIII, vol. 6237 of Proceedings of SPIE, pp. 1-8, Orlando, Fla, USA, April 2006.

[8] D. M. Zasada, P. K. Sanyal, and R. P. Perry, "Detecting moving targets in multiple-channel SAR via double thresholding," in Proceedings of the IET International Conference on Radar Systems, Edinburgh, UK, October 2007.

[9] Y. Zhang, A. Hajjari, K. Kim, and B. Himed, "A dual-threshold ATI-SAR approach for detecting slow moving targets," in Proceedings of IEEE International Radar Conference (RADAR '05), pp. 295-299, Washington, DC, USA, May 2005.

[10] C. E. Muehe and M. Labitt, "Displaced-phase-center antenna technique," Lincoln Laboratory Journal, vol. 12, no. 2, pp. 281296, 2000.

[11] J. Ward, "Space-time adaptive processing for airborne radar," Tech. Rep. ESC-TR-94-109, MIT Lincoln Laboratory, Lexington, Mass, USA, 1994.

[12] P. K. Sanyal, "STAP processing monostatic and bistatic MCARM data," Tech. Rep. AFRL-SN-RS-TR-1999-197, MITRE Corporation, Bedford, Mass, USA, 1999. 